\begin{tabular}{|c|c|}
\hline Title & A general ization of the weighted Strichartz estimates for wave equations and an application to self-similar solutions \\
\hline Author(s) & Kato, Jun; Nakamura, Makoto; Ozawa, Tohru \\
\hline Citation & $\begin{array}{l}\text { Communications on Pure and A pplied Mathematics, 60(2), } 164186 \\
\text { https://doi.org/10.1002/cpa.20133 }\end{array}$ \\
\hline Issue Date & 2007-02 \\
\hline Doc URL & http:/hdl.handle.net/2115/17152 \\
\hline Rights & $\begin{array}{l}\text { Copyright @ } 2006 \text { Wiley Periodical s, Inc., Communications on Pure and A pplied Mathematics, volume 60, issue 2, pp. } \\
164186\end{array}$ \\
\hline Type & article (author version) \\
\hline File Information & CPAM60-2.pdf \\
\hline
\end{tabular}

Instructions for use 


\title{
A generalization of the weighted Strichartz estimates for wave equations and an application to self-similar solutions
}

\author{
Jun Kato, Makoto Nakamura and Tohru Ozawa
}

\begin{abstract}
Weighted Strichartz estimates with homogeneous weights with critical exponents are proved for the wave equation without support restriction on the forcing term. The method of proof is based on the expansion by spherical harmonics and on the Sobolev space over the unit sphere, by which the required estimates are reduced to the radial case. As an application of the weighted Strichartz estimates, the existence and uniqueness of self-similar solutions to nonlinear wave equations is proved up to 5 space dimensions.
\end{abstract}

\section{Introduction and main results}

We consider the Cauchy problem of the inhomogeneous wave equation with zero data

$$
\begin{aligned}
& \partial_{t}^{2} w-\Delta w=F, \quad(t, x) \in(0, \infty) \times \mathbb{R}^{n} \equiv \mathbb{R}_{+}^{1+n}, \\
& \left.w\right|_{t=0}=0,\left.\partial_{t} w\right|_{t=0}=0, \quad x \in \mathbb{R}^{n},
\end{aligned}
$$

and the associated weighted Strichartz estimates of the form

$$
\left\|\left.\left.\left|t^{2}-\right| x\right|^{2}\right|^{a} w\right\|_{L^{q}\left(\mathbb{R}_{+}^{1+n}\right)} \leq C\left\|\left.\left.\left|t^{2}-\right| x\right|^{2}\right|^{b} F\right\|_{L^{q^{\prime}\left(\mathbb{R}_{+}^{1+n}\right)}}, \quad 2 \leq q \leq \frac{2(n+1)}{n-1},
$$

where $q^{\prime}$ is the conjugate exponent to $q$, and $a$ and $b$ are to be specified below. Estimates (1.3) are regarded as the hyperbolic version of the following weighted estimates for the Laplacian

$$
\left\|\left.|| x\right|^{a} f\right\|_{L^{q}\left(\mathbb{R}^{n}\right)} \leq C\left|\left\|\left.x\right|^{b} \Delta f\right\|_{L^{q^{i}}\left(\mathbb{R}^{n}\right)}, \quad 2 \leq q \leq \frac{2 n}{n-2} .\right.
$$

See [10], for example.

Estimates (1.3) were proved by Georgiev-Lindblad-Sogge [7] under the following conditions

$$
a<\frac{n-1}{2}-\frac{n}{q}, \quad b>\frac{1}{q}, \quad \operatorname{supp} F \subset\{(t, x) ;|x|<t-1\} .
$$

Using these estimates, they solved part of Strauss' conjecture concerning the global Cauchy problem of nonlinear wave equation with compactly supported, smooth, small initial data. D'Ancona-GeorgievKubo [5] removed the support condition of $F$ in (1.4). Tataru [31] proved (1.3) when

$$
a-b+\frac{n+1}{q}=\frac{n-1}{2}, \quad b<\frac{1}{q}, \quad \operatorname{supp} F \subset\{(t, x) ;|x|<t\},
$$

where the first one is related to the scale invariance. 
The purpose of this paper is to show the estimates (1.3) in the scale invariant case without the support condition on $F$, which have an application to the existence of the self-similar solutions to nonlinear wave equations as we shall see below. In [13, 14], it was shown that the estimates (1.3) hold if $F$ is radial in space variables without the support condition on $F$. Precisely, it was proved that the estimates (1.3) hold if

$$
a-b+\frac{n+1}{q}=\frac{n-1}{2}, \quad \frac{n}{q}-\frac{n-1}{2}<b<\frac{1}{q}, \quad F(t, x)=\widetilde{F}(t,|x|) .
$$

except the borderline cases $q=2,2(n+1) /(n-1)$. As compared with the condition (1.5) the support condition of $F$ is removed at the cost of the additional lower bound on $b$, namely, $b>n / q-(n-1) / 2$.

In this paper, we remove the assumption of radial symmetry on $F$ in (1.6):

Theorem 1.1. Let $n \geq 2$. Let $q$, a, b satisfy $2<q<2(n+1) /(n-1)$,

$$
a-b+\frac{n+1}{q}=\frac{n-1}{2}, \quad \frac{n}{q}-\frac{n-1}{2}<b<\frac{1}{q} .
$$

Then, the solution $w$ to (1.1), (1.2) satisfies the estimate

$$
\left\|| t ^ { 2 } - | x | ^ { 2 } \left|{ }^{a} w\left\|_{L_{t, r}^{q} L_{\omega}^{2}} \leq\left.\left. C||\left|t^{2}-\right| x\right|^{2}\right|^{b} F\right\|_{L_{t, r}^{q^{\prime}} L_{\omega}^{2}}\right.\right.
$$

Here, for $G=G(t, x)$ the norm $\|\cdot\|_{L_{t, r}^{p} L_{\omega}^{2}}$ is defined by

$$
\|G\|_{L_{t, r}^{p} L_{\omega}^{2}}=\left\{\int_{0}^{\infty} \int_{0}^{\infty}\|G(t, r \cdot)\|_{L^{2}\left(S^{n-1}\right)}^{p} r^{n-1} d r d t\right\}^{1 / p}
$$

based on the polar coordinates $x=r \omega, r=|x|>0, \omega=x /|x| \in S^{n-1}$. A novelty in Theorem 1.1 consists in the introduction of $L^{2}$ space on the sphere, which enables us to remove the assumption of radial symmetry on $F$.

In odd space dimensions, we are able to obtain a gain of regularity with respect to angular variables in (1.8).

Theorem 1.2. Let $n \geq 3$ be odd. Let $q, a$, b satisfy $4(n-1) /(2 n-3)<q<2(n+1) /(n-1)$,

$$
a-b+\frac{n+1}{q}=\frac{n-1}{2}, \quad \frac{n+1}{2 q}-\frac{n-1}{4}<b<\frac{1}{q} .
$$

Then, the solution $w$ to (1.1), (1.2) satisfies the estimate

$$
\left\|\left.\left.\left|t^{2}-\right| x\right|^{2}\right|^{a} w\right\|_{L_{t, r}^{q} H_{\omega}^{1 / 2}} \leq C\left|\left\|\left.\left.\left|t^{2}-\right| x\right|^{2}\right|^{b} F\right\|_{L_{t, r}^{q^{\prime}} L_{\omega}^{2}} .\right.
$$

Remark 1.3. The lower bound on $b$ in Theorem 1.2 is strictly greater than the one in Theorem 1.1 for $q>2$.

Remark 1.4. $H_{\omega}^{s}$ denotes the Sobolev space on $S^{n-1}$ of fractional order $s$ and the norm $\|\cdot\|_{L_{t, r}^{q} H_{\omega}^{s}}$ is defined analogously to (1.9). See the appendix below.

The idea of the proof of Theorems 1.1, 1.2 is based on the expansion by spherical harmonics. We derive the expansion of the solution $w$ with respect to spherical harmonics and reduce the estimates essentially to radial case. This idea is due to [20], which treats end point Strichartz estimates for the 
wave equation in three space dimensions by using the norm with respect to angular variables. We also notice that a similar type of Strichartz estimates are treated in [19]. See also [3].

This paper is organized as follows. In Section 2 we prove Theorems 1.1, 1.2. In Section 3 we give an application of these theorems to the existence of self-similar solutions to nonlinear wave equations. In Appendix we summarize basic properties of Sobolev spaces over the unit sphere.

\section{Proof of the theorems}

The proofs of Theorems 1.1, 1.2 are based on the expansion of the solution $w$ with respect to the spherical harmonics. We first describe its expansion precisely.

For $k \geq 0$, We denote by $\mathscr{H}_{k}$ the space of spherical harmonics of degree $k$ on $S^{n-1}$, by $\alpha_{k}$ its dimension, and by $\left\{Y_{1}^{k}, \cdots, Y_{\alpha_{k}}^{k}\right\}$ the orthonormal basis of $\mathscr{H}_{k}$. It is well known that $L^{2}\left(S^{n-1}\right)=\oplus_{k=0}^{\infty} \mathscr{H}_{k}$ and that $F(t, x)=F(t, r \omega)$ has the expansion

$$
F(t, r \omega)=\sum_{k=0}^{\infty} \sum_{l=1}^{\alpha_{k}} F_{l}^{k}(t, r) Y_{l}^{k}(\omega)
$$

Then, by orthogonality, we observe that $\|F(t, r \cdot)\|_{L^{2}\left(S^{n-1}\right)}=\left(\sum_{k, l}\left|F_{l}^{k}(t, r)\right|^{2}\right)^{1 / 2}$ and more generally,

$$
\|F(t, r \cdot)\|_{H^{s}\left(S^{n-1}\right)}=\left\{\sum_{k, l}(1+k(k+n-2))^{s}\left|F_{l}^{k}(t, r)\right|^{2}\right\}^{1 / 2} .
$$

Note that $\left(-\Delta_{S^{n-1}}\right) Y^{k}=k(k+n-2) Y^{k}$ for $Y^{k} \in \mathscr{H}_{k}$, where $\Delta_{S^{n-1}}$ is the Laplace-Beltrami operator on $S^{n-1}$.

In the following, we set

$$
W_{n}(t)=(-\Delta)^{-1 / 2} \sin \left[t(-\Delta)^{1 / 2}\right],
$$

where we specially affix the space dimension $n$ for later purpose (see Lemma 2.1 below). Then, the solution $w$ to (1.1), (1.2) is given by

$$
w(t, r \omega)=\int_{0}^{t}\left[W_{n}(t-s) F(s, \cdot)\right](r \omega) d s,
$$

which is written in terms of (2.1) by

$$
w(t, r \omega)=\sum_{k=0}^{\infty} \sum_{l=1}^{\alpha_{k}} \int_{0}^{t}\left[W_{n}(t-s)\left\{F_{l}^{k}(s, \lambda) Y_{l}^{k}(\theta)\right\}\right](r \omega) d s .
$$

Then, we use the following lemma.

Lemma 2.1. Let $Y^{k} \in \mathscr{H}_{k}$. Then, for $f \in C_{0}^{\infty}((0, \infty))$,

$$
W_{n}(t)\left[f(\lambda) Y^{k}(\theta)\right](r \omega)=r^{k} W_{n+2 k}(t)\left[\lambda^{-k} f(\lambda)\right](r) Y^{k}(\omega) .
$$

Remark 2.2. We apply Lemma 2.1 to compute (2.3). To prove Theorems 1.1, 1.2 it suffices to show for $F \in C_{0}^{\infty}\left(\mathbb{R}_{+}^{1+n} \backslash\{|x|=0\}\right)$. In fact, such space is dense in the weighted Lebesgue spaces in question, and then, for each $t \geq 0$ the function

$$
r \mapsto F_{l}^{k}(t, r)=\int_{S^{n-1}} F(t, r \theta) Y_{l}^{k}(\theta) d \sigma(\theta)
$$


is smooth with compact support. Note that since $F \in C_{0}^{\infty}\left(\mathbb{R}_{+}^{1+n} \backslash\{|x|=0\}\right), F_{l}^{k}(t, r)$ vanishes when $r$ is sufficiently small.

Proof of Lemma 2.1. Since $f \in C_{0}^{\infty}((0, \infty))$, the left hand side of $(2.4)$ is a classical solution of the Cauchy problem of the wave equation

$$
\begin{aligned}
& \partial_{t}^{2} v-\Delta v=0 \\
& v(0, x)=0, \quad \partial_{t} v(0, x)=f(|x|) Y^{k}(x /|x|)
\end{aligned}
$$

Thus, if we show that the right hand side of (2.4), defined by

$$
z(t, r \omega)=r^{k} \widetilde{z}(t, r) Y^{k}(\omega), \quad \widetilde{z}(t, r)=W_{n+2 k}(t)\left[\lambda^{-k} f(\lambda)\right](r),
$$

is also a classical solution of (2.5), (2.6), then by the uniqueness of classical solutions we obtain (2.4). Obviously, $z$ is regular and satisfies (2.6). Therefore, it suffices to show that $z$ satisfies (2.5), which follows from

$$
\begin{aligned}
& \left(\partial_{t}^{2}-\Delta\right) z \\
= & \left(\partial_{t}^{2}-\partial_{r}^{2}-\frac{n-1}{r} \partial_{r}-\frac{1}{r^{2}} \Delta_{S^{n-1}}\right) r^{k} \widetilde{z} Y^{k} \\
= & r^{k}\left(\partial_{t}^{2} \widetilde{z}-\partial_{r}^{2} \widetilde{z}-\frac{n+2 k-1}{r} \partial_{r} \widetilde{z}-\frac{k(k+n-2)}{r^{2}} \widetilde{z}\right) Y^{k}+r^{k} \widetilde{z} \frac{k(k+n-2)}{r^{2}} Y^{k} \\
= & r^{k}\left(\partial_{t}^{2} \widetilde{z}-\partial_{r}^{2} \widetilde{z}-\frac{n+2 k-1}{r} \partial_{r} \widetilde{z}\right) Y^{k}=0 .
\end{aligned}
$$

This completes the proof of Lemma 2.1 .

Applying Lemma 2.1, we obtain the expansion of $w$

$$
w(t, r \omega)=\sum_{k=0}^{\infty} \sum_{l=1}^{\alpha_{k}} S_{k}\left(F_{l}^{k}\right)(t, r) Y_{l}^{k}(\omega),
$$

where

$$
S_{k}(G)(t, r)=r^{k} \int_{0}^{t} W_{n+2 k}(t-s)\left[\lambda^{-k} G(s, \lambda)\right](r) d s .
$$

Using this expansion, we prove Theorems 1.1, 1.2.

Proof of Theorem 1.1. By the expansion (2.7), a crucial point of the proof of Theorem 1.1 is to derive the estimate on the coefficients $S_{k}\left(F_{l}^{k}\right)$, which is derived by a similar argument in $[13,14]$, where the weighted Strichartz estimates under the assumption of radial symmetry are considered. In particular, the following estimates hold.

Lemma 2.3. Let $n \geq 2$. Let $q$, $a$, and $b$ be as in Theorem 1.1. Then, there exists a constant $C>0$ independent of $k$ such that

$$
\left\|\left|t^{2}-r^{2}\right|^{a} r^{(n-1) / q} S_{k}(G)\right\|_{L_{t, r}^{q}\left(\mathbb{R}_{+}^{2}\right)} \leq C\left\||| t^{2}-\left.r^{2}\right|^{b} r^{(n-1) / q^{\prime}} G\right\|_{L_{t, r}^{q^{\prime}}\left(\mathbb{R}_{+}^{2}\right)} .
$$


Proof of Lemma 2.3. We first consider the case where the space dimension $n$ is odd. From (2.8) and the representation of the radial solution (see for instance [30, Lemma 2.2]), we have

$$
S_{k}(G)(t, r)=r^{-(n-1) / 2} \int_{0}^{t} \int_{|t-s-r|}^{t-s+r} P_{k+(n-3) / 2}(\mu) \lambda^{(n-1) / 2} G(s, \lambda) d \lambda d s,
$$

where $P_{m}$ is the Legendre polynomial of degree $m$ and

$$
\mu=\frac{r^{2}+\lambda^{2}-(t-s)^{2}}{2 r \lambda} .
$$

Then, from the estimate of the Legendre polynomials

$$
\left|P_{m}(z)\right| \leq 1, \quad|z| \leq 1, m \geq 0
$$

and the fact that $|\mu| \leq 1$ if $\lambda \geq|t-s-r|$, we estimate

$$
\left|S_{k}(G)(t, r)\right| \leq r^{-(n-1) / 2} \int_{0}^{t} \int_{|t-s-r|}^{t-s+r} \lambda^{(n-1) / 2}|G(s, \lambda)| d \lambda d s .
$$

Thus, to derive the estimate (2.9) it is sufficient to apply the same argument as in [13, Lemma 3.3]. Note that the right hand side of (2.13) is independent of $k$.

We next consider the case where $n$ is even. In this case we need two types of representations and estimates of $S_{k}(G)(t, r)$ to apply the argument in [14]. From (2.8) and the representation of the radial solution (see for instance [30, Lemma 2.3]), we have

$$
\begin{aligned}
S_{k}(G)(t, r) & =\frac{2}{\pi} r^{-n / 2+1} \int_{0}^{t} \int_{0}^{t-s} \frac{\rho}{\sqrt{(t-s)^{2}-\rho^{2}}} \\
& \times\left(\int_{|r-\rho|}^{r+\rho} \frac{T_{k+(n-2) / 2}(\widetilde{\mu})}{\sqrt{\lambda^{2}-(r-\rho)^{2}} \sqrt{(r+\rho)^{2}-\lambda^{2}}} \lambda^{n / 2} G(s, \lambda) d \lambda\right) d \rho d s,
\end{aligned}
$$

where $T_{m}$ is the Tchebysheff polynomial of degree $m$ and $\tilde{\mu}=\left(\lambda^{2}+r^{2}-\rho^{2}\right) / 2 r \lambda$. Since $\left|T_{m}(z)\right| \leq 1$ for $|z| \leq 1, m \geq 0$, and $|\widetilde{\mu}| \leq 1$ for $\lambda \geq|r-\rho|$, we obtain the pointwise estimate of $S_{k}(G)(t, r)$ independent of $k$. Similarly, from (2.8) and the representation of radial solution (see for instance [18, Theorem 3.4]), we have

$$
\begin{array}{r}
S_{k}(G)(t, r)=r^{-k-n+2} \int_{0}^{t} \int_{|t-s-r|}^{t-s+r} \lambda^{k+n-1} K_{k+(n-2) / 2}(\lambda, r, t-s) G(s, \lambda) d \lambda d s \\
\quad+r^{-k-n+2} \int_{0}^{\max (t-r, 0)} \int_{0}^{t-s-r} \lambda^{k+n-1} \widetilde{K}_{k+(n-2) / 2}(\lambda, r, t-s) G(s, \lambda) d \lambda d s .
\end{array}
$$

Here the kernels have the estimates (see [18, Lemma 4.2], [14, Lemma 3.1])

$$
\begin{gathered}
r^{-k} \lambda^{k}\left|K_{k+(n-2) / 2}(\lambda, r, \tau)\right| \leq C r^{(n-3) / 2} \lambda^{-(n-1) / 2} \min \left(r^{1 / 2}, \lambda^{1 / 2}\right)(\lambda-\tau+r)^{-1 / 2}, \\
|\tau-r|<\lambda<\tau+r, \\
r^{-k} \lambda^{k}\left|\widetilde{K}_{k+(n-2) / 2}(\lambda, r, \tau)\right| \leq C r^{(n-3) / 2+\sigma}(\tau-r)^{-(n-2) / 2-\sigma}(\tau-r-\lambda)^{-1 / 2}, \\
0<\lambda<\tau-r, \quad 0 \leq \sigma \leq 1 / 2,
\end{gathered}
$$

where the constants are independent of $k$. These representations and estimates of $S_{k}(G)(t, r)$ enable us to apply the argument in [14] to derive the estimate (2.9). 
Then, the estimate (1.8) is obtained as follows. By the expansion (2.7) and Lemma 2.3, we have

$$
\begin{aligned}
\left\|\left.\left|t^{2}-\right| x\right|^{2} \mid{ }^{a} w\right\|_{L_{t, r}^{q} L_{\omega}^{2}} & =\left\|\left|t^{2}-r^{2}\right|^{a} r^{(n-1) / q}\left(\sum_{k, l}\left|S_{k}\left(F_{l}^{k}\right)\right|^{2}\right)^{1 / 2}\right\|_{L_{t, r}^{q}} \\
& \leq\left(\sum_{k, l}\left\|\left|t^{2}-r^{2}\right|^{a} r^{(n-1) / q} S_{k}\left(F_{l}^{k}\right)\right\|_{L_{t, r}^{q}}^{2}\right)^{1 / 2} \\
& \leq C\left(\sum_{k, l}\left\|\left|t^{2}-r^{2}\right|^{b} r^{(n-1) / q^{\prime}} F_{l}^{k}\right\|_{L_{t, r}^{q^{\prime}}}^{2}\right)^{1 / 2} \\
& \leq C\left\|\left|t^{2}-r^{2}\right|^{b} r^{(n-1) / q^{\prime}}\left(\sum_{k, l}\left|F_{l}^{k}\right|^{2}\right)^{1 / 2}\right\|_{L_{t, r}^{q^{\prime}}} \\
& \leq C\left\|\left.\left.\left|t^{2}-\right| x\right|^{2}\right|^{b} F\right\|_{L_{t, r}^{q^{\prime}} L_{\omega}^{2}}
\end{aligned}
$$

where we have used Minkowski's integral inequality repeatedly, since $q>2$ and $q^{\prime}<2$.

Proof of Theorem 1.2. For the proof of Theorem 1.2, we need improved estimates on $S_{k}\left(F_{l}^{k}\right)$ instead of those in Lemma 2.3 and such estimates are derived at least in odd space dimensions.

Lemma 2.4. Let $n \geq 3$ be odd. Let $q, a$, and $b$ be as in Theorem 1.2. Then, there exists a constant $C>0$ independent of $k \geq 1$ such that

$$
\left\|\left|t^{2}-r^{2}\right|^{a} r^{(n-1) / q} S_{k}(G)\right\|_{L_{t, r}^{q}} \leq C k^{-1 / 2}\left\|\left|t^{2}-r^{2}\right|^{b} r^{(n-1) / q^{\prime}} G\right\|_{L_{t, r}^{q^{\prime}}}
$$

Given Lemma 2.4, we obtain (1.11) as follows. By the expansion (2.7) and (2.2), we have

$$
\begin{aligned}
\left\|\left.\left.\left|t^{2}-\right| x\right|^{2}\right|^{a} w\right\|_{L_{t, r}^{q} H_{\omega}^{1 / 2}} & =\left\|t^{2}-\left.r^{2}\right|^{a} r^{(n-1) / q}\left\{\sum_{k, l}(1+k(k+n-2))^{1 / 2}\left|S_{k}\left(F_{l}^{k}\right)\right|^{2}\right\}^{1 / 2}\right\|_{L_{t, r}^{q}} \\
& \leq C\left(\sum_{k, l}(1+k)\left\|\left|t^{2}-r^{2}\right|^{a} r^{(n-1) / q} S_{k}\left(F_{l}^{k}\right)\right\|_{L_{t, r}^{q}}^{2}\right)^{1 / 2} \\
& \leq C\left(\sum_{k, l}\left\|\left|t^{2}-r^{2}\right|^{b} r^{(n-1) / q^{\prime}} F_{l}^{k}\right\|_{L_{t, r}^{q^{\prime}}}^{2}\right)^{1 / 2} \\
& \leq C\left\||| t^{2}-\left.r^{2}\right|^{b} r^{(n-1) / q^{\prime}}\left(\sum_{k, l}\left|F_{l}^{k}\right|^{2}\right)^{1 / 2}\right\|_{L_{t, r}^{q^{\prime}}} \\
& \leq C\left\|\left.\left.\left|t^{2}-\right| x\right|^{2}\right|^{b} F\right\|_{L_{t, r}^{q^{\prime}} L_{\omega}^{2}}
\end{aligned}
$$

where we have used Minkowski's integral inequality repeatedly, since $q>2$ and $q^{\prime}<2$.

The rest of this section is devoted to the proof of Lemma 2.4. As in the radial case $[13,14]$, to prove (2.14) we use the following weighted Hardy-Littlewood-Sobolev inequality.

Lemma 2.5 ([28]). Let $0<\lambda<n, 1<r, s<\infty$. Let $\alpha<n / s^{\prime}$ and $\beta<n / r^{\prime}$ with $\alpha+\beta \geq 0$ satisfy $1 / s+1 / r+(\lambda+\alpha+\beta) / n=2$. Then,

$$
\left|\int_{\mathbb{R}^{n}} \int_{\mathbb{R}^{n}} \frac{f(x) g(y)}{|x|^{\alpha}|x-y|^{\lambda}|y|^{\beta}} d x d y\right| \leq C|| f\left\|_{L^{s}\left(\mathbb{R}^{n}\right)}|| g\right\|_{L^{r}\left(\mathbb{R}^{n}\right)} .
$$


Proof of Lemma 2.4. We recall that $S_{k}(G)$ is given by (2.10) in odd space dimensions. To derive the estimate (2.14) we use another estimate of the Legendre polynomials instead of (2.12). Namely,

$$
\left|P_{m}(z)\right| \leq C m^{-1 / 2}\left(1-|z|^{2}\right)^{-1 / 4}, \quad|z|<1, m \geq 1 .
$$

(See $[6, \S 1.6]$.$) Then, from (2.10)$, we have

$$
\begin{aligned}
& \left|S_{k}(G)(t, r)\right| \\
& \leq C k^{-1 / 2} r^{-(n-1) / 2} \int_{0}^{t} \int_{|t-s-r|}^{t-s+r}\left(1-\mu^{2}\right)^{-1 / 4} \lambda^{(n-1) / 2}|G(s, \lambda)| d \lambda d s .
\end{aligned}
$$

Note that $\mu$ is given by (2.11), and thus

$$
\begin{aligned}
& \frac{\left(1-\mu^{2}\right)^{-1 / 4}}{(r+\lambda+t-s)^{1 / 4}(r+\lambda-t+s)^{1 / 4}(t-s+r-\lambda)^{1 / 4}(t-s-r+\lambda)^{1 / 4}} . \\
& \sqrt{2} r^{1 / 2} \lambda^{1 / 2}
\end{aligned}
$$

To prove (2.14) it suffices to show that

$$
\begin{aligned}
\left|\int_{0}^{\infty} \int_{0}^{\infty}\right| t^{2}-\left.r^{2}\right|^{a} r^{(n-1) / q} & S_{k}(G)(t, r) \Phi(t, r) d r d t \mid \\
& \leq C k^{-1 / 2}\left|\left\|t^{2}-\left.r^{2}\right|^{b} r^{(n-1) / q^{\prime}} G\right\|_{L^{q^{\prime}}}\|\Phi\|_{L^{q^{\prime}}}\right.
\end{aligned}
$$

for all $\Phi \in C_{0}^{\infty}((0, \infty) \times(0, \infty))$ by duality. From (2.16) the left hand side of (2.17) is bounded by the constant multiple of

$$
\begin{aligned}
& k^{-1 / 2} \int_{0}^{\infty} \int_{0}^{\infty} \int_{0}^{t} \int_{|t-s-r|}^{t-s+r}\left|t^{2}-r^{2}\right|^{a} r^{-\delta} \lambda^{(n-1) / 2} \\
& \quad \times\left(1-\mu^{2}\right)^{-1 / 4}|G(s, \lambda)||\Phi(t, r)| d \lambda d s d r d t \\
& =k^{-1 / 2} \int_{0}^{\infty} \int_{0}^{\infty} \int_{0}^{t} \int_{|t-s-r|}^{t-s+r} \frac{\left(\left|s^{2}-\lambda^{2}\right|^{b} \lambda^{(n-1) / q^{\prime}}|G(s, \lambda)|\right)|\Phi(t, r)|}{\left|t^{2}-r^{2}\right|^{-a} r^{\delta}\left|s^{2}-\lambda^{2}\right|^{b} \lambda^{\delta}\left(1-\mu^{2}\right)^{1 / 4}} d \lambda d s d r d t,
\end{aligned}
$$

where $\delta=(n-1)(1 / 2-1 / q)$. Then, the range of $q$ and the equality in (1.10) are rewritten respectively as

$$
\frac{1}{4}<\delta<\frac{n-1}{n+1}, \quad a-b-\delta+\frac{2}{q}=0 .
$$

Applying the change of variables

$$
u=t+r, v=t-r, \xi=s+\lambda, \eta=s-\lambda,
$$

and the substitutions

$$
\left|s^{2}-\lambda^{2}\right|^{b} \lambda^{(n-1) / q^{\prime}}|G(s, \lambda)| \equiv H(\xi, \eta), \quad|\Phi(t, r)| \equiv \Psi(u, v),
$$

we see that the right hand side of (2.18) equals to

$$
\begin{aligned}
k^{-1 / 2}\left(\int_{0}^{\infty} \int_{0}^{u} \int_{v}^{u} \int_{-\xi}^{v}+\right. & \left.\int_{0}^{\infty} \int_{-u}^{0} \int_{-v}^{u} \int_{-\xi}^{v}\right) \\
& \times \frac{H(\xi, \eta) \Psi(u, v)}{|u|^{-a}|v|^{-a}|u-v|^{\delta-1 / 2}|\xi|^{b}|\eta|^{b}|\xi-\eta|^{\delta-1 / 2}} \\
& \times \frac{d \eta d \xi d v d u}{|u-\eta|^{1 / 4}|\xi-v|^{1 / 4}|u-\xi|^{1 / 4}|v-\eta|^{1 / 4}}
\end{aligned}
$$


Changing the order of the integrals, we see that (2.20) equals to

$$
k^{-1 / 2} \int_{-\infty}^{\infty} \int_{|v|}^{\infty} \int_{\xi}^{\infty} \int_{-\xi}^{v} \cdots d \eta d u d \xi d v
$$

which is divided into two parts as

$$
k^{-1 / 2} \int_{-\infty}^{\infty} \int_{|v|}^{\infty}\left(\int_{\xi}^{2 \xi} \int_{-\xi}^{v}+\int_{2 \xi}^{\infty} \int_{-\xi}^{v}\right) \cdots d \eta d u d \xi d v \equiv k^{-1 / 2}\left(I_{1}+I_{2}\right)
$$

We notice that in both of the domains of integration above, the condition

$$
-\xi \leq \eta \leq v \leq|v| \leq \xi \leq u
$$

holds.

We first consider the case where $\delta \geq 1 / 2$, i.e. $n \geq 5$ and $2(n-1) /(n-2) \leq q<2(n+1) /(n-1)$.

Estimate of $I_{1}$. Since $\delta \geq 1 / 2$ and $\eta \leq v \leq \xi \leq u$, we have

$$
\begin{aligned}
& |u-v|^{-\delta+1 / 2} \leq|u-\xi|^{-\delta / 2+1 / 4}|\xi-v|^{-\delta / 2+1 / 4} \\
& |\xi-\eta|^{-\delta+1 / 2} \leq|\xi-v|^{-\delta / 2+1 / 4}|v-\eta|^{-\delta / 2+1 / 4}
\end{aligned}
$$

Combining the above estimates with $|u-\eta|^{-1 / 4} \leq|\xi-v|^{-1 / 4}$, we obtain

$$
I_{1} \leq \int_{-\infty}^{\infty} \int_{|v|}^{\infty} \frac{1}{|v|^{-a}|\xi-v|^{\delta}|\xi|^{b}}\left(\int_{\xi}^{2 \xi} \int_{-\xi}^{v} \frac{H(\xi, \eta) \Psi(u, v)}{|u|^{-a}|u-\xi|^{\delta / 2}|v-\eta|^{\delta / 2}|\eta|^{b}} d \eta d u\right) d \xi d v
$$

Then, we compute the inner integral as

$$
\begin{aligned}
& \int_{\xi}^{2 \xi} \int_{-\xi}^{v} \frac{H(\xi, \eta) \Psi(u, v)}{|u|^{-a}|u-\xi|^{\delta / 2}|v-\eta|^{\delta / 2}|\eta|^{b}} d \eta d u \\
& =\left(\int_{\xi}^{2 \xi} \frac{\Psi(u, v)}{|u|^{-a}|u-\xi|^{\delta / 2}} d u\right)\left(\int_{-\xi}^{v} \frac{H(\xi, \eta)}{|v-\eta|^{\delta / 2}|\eta|^{b}} d \eta\right) \\
& \leq\left(\int_{\xi}^{2 \xi} \frac{d u}{|u|^{-a q}|u-\xi|^{q \delta / 2}}\right)^{1 / q}\|\Psi(\cdot, v)\|_{L^{q^{\prime}}}\left(\int_{-\infty}^{v} \frac{d \eta}{|v-\eta|^{q \delta / 2}|\eta|^{b q}}\right)^{1 / q}\|H(\xi, \cdot)\|_{L^{q^{\prime}}} \\
& =C|\xi|^{a-\delta / 2+1 / q}|| \Psi(\cdot, v)\left\|_{L^{q^{\prime}}}|v|^{-\delta / 2-b+1 / q}|| H(\xi, \cdot)\right\|_{L^{q^{\prime}}}
\end{aligned}
$$

We notice that the above integrals converge, since $q \delta / 2<1, b q<1$, and $q \delta / 2+b q>1$. In fact, $q \delta / 2<1$ is equivalent to $q<2(n+1) /(n-1)$, and $q \delta / 2+b q>1$ is equivalent to $b>(n+1) / 2 q-(n-1) / 4$, since $\delta=(n-1)(1 / 2-1 / q)$. Thus, we obtain

$$
I_{1} \leq C \int_{-\infty}^{\infty} \int_{-\infty}^{\infty} \frac{\|\Psi(\cdot, v)\|_{L^{q^{\prime}}}|| H(\xi, \cdot) \|_{L^{q^{\prime}}}}{|v|^{-a+b+\delta / 2-1 / q}|\xi-v|^{\delta}|\xi|^{-a+b+\delta / 2-1 / q}} d \xi d v .
$$

From (1.10), (2.19) we observe that

$$
\begin{aligned}
& 0<-a+b+\frac{\delta}{2}-\frac{1}{q}=\frac{n+1}{2 q}-\frac{n-1}{4}<\frac{1}{q}, \\
& \frac{2}{q^{\prime}}+\left(\delta+2\left(-a+b+\frac{\delta}{2}-\frac{1}{q}\right)\right)=2,
\end{aligned}
$$


which enable us to apply Lemma 2.5 to get

$$
I_{1} \leq C|| \Psi\left\|_{L^{q^{\prime}}}\right\| H\left\|_{L^{q^{\prime}}}=C\left|\left\|t^{2}-\left.r^{2}\right|^{b} r^{(n-1) / q^{\prime}} G\right\|_{L^{q^{\prime}}}\right| \mid \Phi\right\|_{L^{q^{\prime}}} .
$$

Estimate of $I_{2}$. Since $\delta \geq 1 / 2$ and $\eta \leq v \leq \xi \leq u$, we have

$$
\begin{aligned}
& |u-v|^{-\delta+1 / 2} \leq|u-\xi|^{-\delta+1 / 2}, \\
& |\xi-\eta|^{-\delta+1 / 2} \leq|\xi-v|^{-\delta / 2+1 / 4}|v-\eta|^{-\delta / 2+1 / 4} .
\end{aligned}
$$

Combining the above estimates with $|u-\eta|^{-1 / 4} \leq|u-\xi|^{-1 / 4}$, we obtain

$$
I_{2} \leq \int_{-\infty}^{\infty} \int_{|v|}^{\infty} \frac{1}{|v|^{-a}|\xi-v|^{\delta / 2}|\xi|^{b}}\left(\int_{2 \xi}^{\infty} \int_{-\xi}^{v} \frac{H(\xi, \eta) \Psi(u, v)}{|u|^{-a}|u-\xi|^{\delta}|v-\eta|^{\delta / 2}|\eta|^{b}} d \eta d u\right) d \xi d v .
$$

In the same way as above, we compute the inner integral as

$$
\begin{aligned}
& \int_{2 \xi}^{\infty} \int_{-\xi}^{v} \frac{H(\xi, \eta) \Psi(u, v)}{|u|^{-a}|u-\xi|^{\delta}|v-\eta|^{\delta / 2}|\eta|^{b}} d \eta d u \\
& \leq\left(\int_{2 \xi}^{\infty} \frac{d u}{|u|^{-a q}|u-\xi|^{q \delta}}\right)^{1 / q}\|\Psi(\cdot, v)\|_{L^{q^{\prime}}}\left(\int_{-\infty}^{v} \frac{d \eta}{|v-\eta|^{q \delta / 2}|\eta|^{b q}}\right)^{1 / q}\|H(\xi, \cdot)\|_{L^{q^{\prime}}} \\
& =C|\xi|^{a-\delta+1 / q}|v|^{-\delta / 2-b+1 / q} \mid \Psi(\cdot, v)\left\|_{L^{q^{\prime}}}\right\| H(\xi, \cdot) \|_{L^{q^{\prime}}} .
\end{aligned}
$$

We notice that the above integrals converge, since $-a q+q \delta>1$ and $q \delta / 2<1$. Note that $-a q+q \delta>1$ is equivalent to $b<1 / q$. Since $|v|<\xi$, we observe that $1 /|\xi|^{\delta / 2} \leq(2 /|\xi-v|)^{\delta / 2}$, and thus we obtain

$$
I_{2} \leq C \int_{-\infty}^{\infty} \int_{-\infty}^{\infty} \frac{\|\Psi(\cdot, v)\|_{L^{q^{\prime}}}|| H(\xi, \cdot) \|_{L^{q^{\prime}}}}{|v|^{-a+b+\delta / 2-1 / q}|\xi-v|^{\delta}|\xi|^{-a+b+\delta / 2-1 / q}} d \xi d v
$$

which is the same bound as $I_{1}$. Therefore, applying Lemma 2.5 , we obtain

$$
I_{2} \leq C|| \Psi\left\|_{L^{q^{\prime}}}\right\| H\left\|_{L^{q^{\prime}}}=C\right\||| t^{2}-\left.r^{2}\right|^{b} r^{(n-1) / q^{\prime}} G\left\|_{L^{q^{\prime}}}\right\| \Phi \|_{L^{q^{\prime}}}
$$

This completes the proof in the case $\delta \geq 1 / 2$.

We next consider the case where $1 / 4<\delta<1 / 2$, i.e. $4(n-1) /(2 n-3)<q<2(n-1) /(n-2)$. Since $\delta<1 / 2$ and $|v| \leq u,|\eta| \leq \xi$ (Recall that (2.21)), we have

$$
|u-v|^{-\delta+1 / 2} \leq 2|u|^{-\delta+1 / 2}, \quad|\xi-\eta|^{-\delta+1 / 2} \leq 2|\xi|^{-\delta+1 / 2} .
$$

Estimate of $I_{1}$. Combining the estimate $|u-\eta|^{-1 / 4} \leq|\xi-v|^{-1 / 4}$ with (2.22), we obtain

$$
\begin{aligned}
I_{1} \leq 4 \int_{-\infty}^{\infty} \int_{|v|}^{\infty} \frac{1}{|v|^{-a}|\xi-v|^{1 / 2}|\xi|^{b+\delta-1 / 2}} & \\
& \times\left(\int_{\xi}^{2 \xi} \int_{-\xi}^{v} \frac{H(\xi, \eta) \Psi(u, v)}{|u|^{-a+\delta-1 / 2}|u-\xi|^{1 / 4}|v-\eta|^{1 / 4}|\eta|^{b}} d \eta d u\right) d \xi d v
\end{aligned}
$$


As in the previous case, we compute the inner integral as

$$
\begin{aligned}
& \int_{\xi}^{2 \xi} \int_{-\xi}^{v} \frac{H(\xi, \eta) \Psi(u, v)}{|u|^{-a+\delta-1 / 2}|u-\xi|^{1 / 4}|v-\eta|^{1 / 4}|\eta|^{b}} d \eta d u \\
& \leq\left(\int_{\xi}^{2 \xi} \frac{d u}{|u|^{-a q+q \delta-q / 2}|u-\xi|^{q / 4}}\right)^{1 / q}\|\Psi(\cdot, v)\|_{L^{q^{\prime}}}\left(\int_{-\infty}^{v} \frac{d \eta}{|v-\eta|^{q / 4}|\eta|^{b q}}\right)^{1 / q}\|H(\xi, \cdot)\|_{L^{q^{\prime}}} \\
& =C|\xi|^{a-\delta+1 / 4+1 / q}|v|^{-1 / 4-b+1 / q}|| \Psi(\cdot, v)\left\|_{L^{q^{\prime}}}\right\| H(\xi, \cdot) \|_{L^{q^{\prime}}} .
\end{aligned}
$$

We notice that the above integrals converge, since $q<4$, and $q / 4+b q>1$. Here, the condition $q<4$ is valid if $n \geq 3$ and $q<2(n+1) /(n-1)$, and $q / 4+b q>1$ is also valid if $b>(n+1) / 2 q-(n-1) / 4$ as long as $q<2(n-1) /(n-2)$. Thus,

$$
I_{1} \leq C \int_{-\infty}^{\infty} \int_{-\infty}^{\infty} \frac{\|\Psi(\cdot, v)\|_{L^{q^{\prime}}}\|H(\xi, \cdot)\|_{L^{q^{\prime}}}}{|v|^{-a+b-1 / q+1 / 4}|\xi-v|^{1 / 2}|\xi|^{-a+b+2 \delta-1 / q-3 / 4}} d \xi d v
$$

Here, the condition of Lemma $2.5-a+b-1 / q+1 / 4<1 / q$ is equivalent to $q>4(n-1) /(2 n-3)$, $-a+b+2 \delta-1 / q-3 / 4<1 / q$ is equivalent to $\delta<3 / 4$, and $(-a+b-1 / q+1 / 4)+(-a+b+2 \delta-$ $1 / q-3 / 4)=2 / q-1 / 2>0$. Therefore, applying Lemma 2.5 , we obtain

$$
I_{1} \leq C|| \Psi\left\|_{L^{q^{\prime}}}\right\| H\left\|_{L^{q^{\prime}}}=C\left|\left\|t^{2}-\left.r^{2}\right|^{b} r^{(n-1) / q^{\prime}} G\right\|_{L^{q^{\prime}}}\|\Phi\|_{L^{q^{\prime}}}\right.\right.
$$

Estimate of $I_{2}$. Combining the estimate $|u-\eta|^{-1 / 4} \leq|u-\xi|^{-1 / 4}$ with (2.22), we obtain

$$
\begin{aligned}
I_{2} \leq 4 \int_{-\infty}^{\infty} \int_{|v|}^{\infty} \frac{1}{|v|^{-a}|\xi-v|^{1 / 4}|\xi|^{b+\delta-1 / 2}} & \\
& \times\left(\int_{2 \xi}^{\infty} \int_{-\xi}^{v} \frac{H(\xi, \eta) \Psi(u, v)}{|u|^{-a+\delta-1 / 2}|u-\xi|^{1 / 2}|v-\eta|^{1 / 4}|\eta|^{b}} d \eta d u\right) d \xi d v .
\end{aligned}
$$

As in the previous case, we compute the inner integral as

$$
\begin{aligned}
& \int_{2 \xi}^{\infty} \int_{-\xi}^{v} \frac{H(\xi, \eta) \Psi(u, v)}{|u|^{-a+\delta-1 / 2}|u-\xi|^{1 / 2}|v-\eta|^{1 / 4}|\eta|^{b}} d \eta d u \\
& \leq\left(\int_{2 \xi}^{\infty} \frac{d u}{|u|^{-a q+q \delta-q / 2}|u-\xi|^{q / 2}}\right)^{1 / q}\|\Psi(\cdot, v)\|_{L^{q^{\prime}}}\left(\int_{-\infty}^{v} \frac{d \eta}{|v-\eta|^{q / 4}|\eta|^{b q}}\right)^{1 / q}\|H(\xi, \cdot)\|_{L^{q^{\prime}}} \\
& =C|\xi|^{a-\delta+1 / q}|v|^{-1 / 4-b+1 / q}|| \Psi(\cdot, v)\left\|_{L^{q^{\prime}}}\right\| H(\xi, \cdot) \|_{L^{q^{\prime}}} .
\end{aligned}
$$

We notice that the above integrals converge, since $-a q+q \delta>1, q<4, b<1 / q$, and $q / 4+b q>1$. Note that from (2.19) the condition $-a q+q \delta>1$ is equivalent to $b<1 / q$. Since $|v| \leq \xi$, we estimate $1 /|\xi|^{1 / 4} \leq(2 /|\xi-v|)^{1 / 4}$. Thus,

$$
I_{2} \leq C \int_{-\infty}^{\infty} \int_{-\infty}^{\infty} \frac{\|\Psi(\cdot, v)\|_{L^{q^{\prime}}} \mid H(\xi, \cdot) \|_{L^{q^{\prime}}}}{|v|^{-a+b-1 / q+1 / 4}|\xi-v|^{1 / 2}|\xi|^{-a+b+2 \delta-1 / q-3 / 4}} d \xi d v,
$$

which is the same bound as $I_{1}$. Therefore, applying Lemma 2.5, we obtain

$$
I_{2} \leq C|| \Psi\left\|_{L^{q^{\prime}}}\right\| H\left\|_{L^{q^{\prime}}}=C\left|\left\|t^{2}-\left.r^{2}\right|^{b} r^{(n-1) / q^{\prime}} G\right\|_{L^{q^{\prime}}}\|\Phi\|_{L^{q^{\prime}}}\right.\right.
$$

This completes the proof in the case $1 / 4<\delta<1 / 2$. 


\section{Existence of self-similar solutions}

As an application of Theorems 1.1, 1.2, we show the existence of self-similar solutions to the nonlinear wave equation

$$
\partial_{t}^{2} u-\Delta u=f(u), \quad(t, x) \in \mathbb{R}_{+}^{1+n},
$$

where $n \geq 2, f(u)$ is homogeneous of degree $p>1$ with respect to $u$ and satisfies the estimates

$$
\left|f^{\prime}(u)-f^{\prime}(v)\right| \leq\left\{\begin{array}{l}
C|u-v|^{p-1}, \quad \text { if } 1<p<2 \\
C(|u|+|v|)^{p-2}|u-v|, \quad \text { if } p \geq 2 .
\end{array}\right.
$$

Typical examples of $f(u)$ are given by $\pm|u|^{p}, \pm|u|^{p-1} u$, etc. The solution $u$ to (3.1) is called a self-similar solution if $u$ satisfies

$$
u(t, x)=\lambda^{\frac{2}{p-1}} u(\lambda t, \lambda x)
$$

for all $\lambda>0$. Letting $\lambda=1 / t, u(1, \cdot)=W(\cdot)$, we observe that self-similar solutions are solutions of the form

$$
u(t, x)=t^{-\frac{2}{p-1}} W(x / t) .
$$

From such scaling properties, it is known that self-similar solutions are useful to investigate the asymptotic behavior of the time-global solutions as $t \rightarrow \infty$ (see [21], for example).

It is known that the existence of the self-similar solutions to (3.1) depends heavily on the power $p$ of the nonlinear term. In fact, in three space dimensions, Pecher [25] proved that if $p>1+\sqrt{2}$, there exist self-similar solutions, and if $f(u)=|u|^{p}$ with $p \leq 1+\sqrt{2}$, self-similar solutions do not exist. We intended to extend such sharp existence results of self-similar solutions to higher dimensions. We denote by $p_{0}(n)$ the positive root of

$$
(n-1) p^{2}-(n+1) p-2=0 .
$$

Then, $p_{0}(3)=1+\sqrt{2}$ and we expect $p_{0}(n)$ to be the critical power concerning the existence of selfsimilar solutions to the equation (3.1). We notice that $p_{0}(n)$ is the critical exponent concerning the existence of time-global solutions to the Cauchy problem of the equation (3.1) with small, smooth initial data (see John [11], Georgiev-Lindblad-Sogge [7] and references therein). So, it is natural to expect $p_{0}(n)$ to be the one because self-similar solutions are also time-global solutions.

Concerning this problem, in 2 and 3 space dimensions, Hidano [9] proved the existence of selfsimilar solutions when $p>p_{0}(n)$. In $[13,14]$ the first and third authors proved the existence of radially symmetric self-similar solutions for $p>p_{0}(n)$ with $n \geq 2$.

Remark 3.1. Precisely, the above results show the existence of self-similar solutions for $p_{0}(n)<p<$ $(n+3) /(n-1)$. The existence of self-similar solutions for large $p$ was studied in $[16,24,26]$.

As an application of Theorems 1.1, 1.2, we have the following result.

Theorem 3.2. Let $2 \leq n \leq 5$ and let $p$ satisfy $p_{0}(n)<p<(n+3) /(n-1)$. Suppose $f$ is homogeneous of degree $p$ and satisfy $(\mathrm{H})$. Let $\phi, \psi \in C^{\infty}\left(\mathbb{R}^{n} \backslash\{0\}\right)$ be homogeneous of degree $-2 /(p-1),-2 /(p-$ 1) -1 , respectively. Then, if $\varepsilon>0$ is sufficiently small on the Cauchy data

$$
u(0, x)=\varepsilon \phi(x), \quad \partial_{t} u(0, x)=\varepsilon \psi(x),
$$


there exists a unique global solution u to (3.1) satisfying

$$
\left\|\left.\left.\left|t^{2}-\right| x\right|^{2}\right|^{\gamma} u ; \mathscr{L}_{t, r}^{p+1} H_{\omega}^{(n-1) / 2+\delta}\right\| \leq C \varepsilon
$$

where $\gamma=1 /(p-1)-(n+1) / 2(p+1)$ and $\delta>0$ sufficiently small.

Here, $\mathscr{L}_{t, r}^{q} H_{\omega}^{s}$ denotes the weak Lebesgue spaces on $\mathbb{R}_{+} \times \mathbb{R}_{+}$with values in $H^{s}\left(S^{n-1}\right)$ and $\|\cdot\|_{\mathcal{L}_{t, r}^{q} H_{\omega}^{s}}$ is defined by

$$
\|G\|_{\mathscr{L}_{t, r}^{q} H_{\omega}^{s}} \equiv \sup _{\lambda>0} \lambda\left(\int_{\left.\{(t, r) ; \| G(t, r) \cdot) \|_{H^{s}\left(S^{n-1}\right)}>\lambda\right\}} r^{n-1} d r d t\right)^{1 / q} .
$$

Remark 3.3. By the homogeneity of the data (3.3) and the uniqueness of solutions, solutions obtained in Theorem 3.2 are to be self-similar. That is, self-similar solutions to (3.1) are shown to exist when $2 \leq n \leq 5, p>p_{0}(n)$.

Remark 3.4. Sobolev type embedding theorem on the unit sphere

$$
H^{s}\left(S^{n-1}\right) \hookrightarrow L^{\infty}\left(S^{n-1}\right) \quad \text { for } s>\frac{n-1}{2}
$$

(see (4.3) in Appendix) is basic to our estimates on the nonlinear term, which in turn causes the restriction $n \leq 5$.

The rest of this section is devoted to the proof of Theorem 3.2. For the solution in Theorem 3.2 we mean the solution of the integral equation corresponding to (3.1) with data (3.3),

$$
\begin{aligned}
& u(t)=u_{0}(t)+\int_{0}^{t}(-\Delta)^{-1 / 2} \sin \left[(t-s)(-\Delta)^{1 / 2}\right] f(u(s)) d s \\
& u_{0}(t)=\varepsilon\left(\cos \left[t(-\Delta)^{1 / 2}\right] \phi+(-\Delta)^{-1 / 2} \sin \left[t(-\Delta)^{1 / 2}\right] \psi\right) .
\end{aligned}
$$

We first prepare the following theorem.

Theorem 3.5. Let $n \geq 2$ and let $s \geq 0$. For $(n-1) / 2<\alpha<\min ((n+1) / 2, n-1)$, we assume that $\phi$, $\psi \in C^{\infty}\left(\mathbb{R}^{n} \backslash\{0\}\right)$ are homogeneous of degree $-\alpha,-\alpha-1$, respectively. Then, for $1-(\alpha+2) /(n+1)<$ $1 / q<1-\alpha /(n-1)$, we have

$$
\left.\left.\left|t^{2}-\right| x\right|^{2}\right|^{\gamma} u_{0} \in L_{t, r}^{q} H_{\omega}^{s},
$$

where $\gamma=\alpha / 2-(n+1) / 2 q$.

Proof of Theorem 3.5. For $1 \leq i, j \leq n$, we denote angular derivatives by $\left\{\Omega_{i j}\right\}_{1 \leq i<j \leq n}$ defined by $\Omega_{i j}=$ $x_{i} \partial_{j}-x_{j} \partial_{i}$. For any multi-index $\beta$ with $|\beta| \leq N \equiv n(n-1) / 2$, we define

$$
\Omega^{\beta} \equiv \Omega_{12}^{\beta_{1}} \cdots \Omega_{n-1, n}^{\beta_{N}} .
$$

Then it suffices to show

$$
\left.\left.\left|t^{2}-\right| x\right|^{2}\right|^{\gamma} \Omega^{\beta} u_{0} \in L_{t, r}^{q} L_{\omega}^{2}
$$

for any multi-index $\beta$. Since

$$
\Omega^{\beta} u_{0}(t)=\varepsilon\left(\cos \left[t(-\Delta)^{1 / 2}\right] \Omega^{\beta} \phi+(-\Delta)^{-1 / 2} \sin \left[t(-\Delta)^{1 / 2}\right] \Omega^{\beta} \psi\right),
$$


and $\Omega^{\beta} \phi, \Omega^{\beta} \psi$ are also homogeneous of degree $-\alpha,-\alpha-1$, respectively, we are able to apply [13, Lemma 2.3] to obtain

$$
\left|\Omega^{\beta} u_{0}(t, x)\right| \leq C_{\beta}(t+|x|)^{-\frac{n-1}{2}}|t-| x||^{-\alpha+\frac{n-1}{2}} .
$$

Thus, using polar coordinates $x=r \omega, r>0, \omega \in S^{n-1}$, we have

$$
\left\|\Omega^{\beta} u_{0}(t, r \cdot)\right\|_{L^{2}\left(S^{n-1}\right)} \leq C_{\beta}(t+r)^{-\frac{n-1}{2}}|t-r|^{-\alpha+\frac{n-1}{2}} .
$$

This completes the proof of Theorem 3.5, since the proof of [13, Theorem 2.1] implies the function having the estimate (3.9) satisfy (3.8).

The following theorems are obtained by interpolating the estimates in Theorems 1.1 and 1.2, respectively.

Theorem 3.6. Let $n \geq 2$ and let $s \geq 0$. For $2<q<2(n+1) /(n-1)$ and $(n-1) / q<\alpha<(n-1) / q^{\prime}$, let $a$ and $b$ be defined by

$$
a=\frac{\alpha}{2}-\frac{n+1}{2 q}, \quad b=\frac{\alpha}{2}+\frac{n+1}{2 q}-\frac{n-1}{2} .
$$

Then, there exists a constant $C>0$ such that for any function $F$ which is homogeneous of degree $-\alpha-2$, i.e.

$$
F(\lambda t, \lambda x)=\lambda^{-\alpha-2} F(t, x), \quad(t, x) \in \mathbb{R}_{+}^{1+n}, \lambda>0,
$$

the following estimate holds

$$
\left\|\left.\left.\left|t^{2}-\right| x\right|^{2}\right|^{a} w\right\|_{\mathscr{L}_{t, r}^{q} H_{\omega}^{s}} \leq\left.\left. C||\left|t^{2}-\right| x\right|^{2}\right|^{b} F \|_{\mathscr{L}_{t, r}^{q^{\prime}} H_{\omega}^{s}} .
$$

Theorem 3.7. Let $n \geq 3$ be odd and let $s \geq 1 / 2$. For $4(n-1) /(2 n-3)<q<2(n+1) /(n-1)$ and $(n-1) / 2<\alpha<(n-1) / q^{\prime}$, let $a$ and $b$ as in (3.10). Then, there exists a constant $C>0$ such that for any function $F$ which is homogeneous of degree $-\alpha-2$, the following estimate holds

$$
\left\|| t ^ { 2 } - | x | ^ { 2 } \left|{ } ^ { a } w \left\|_{\mathscr{L}_{t, r}^{q} H_{\omega}^{s}} \leq C\left|\left\|\left.\left|t^{2}-\right| x\right|^{2} \mid{ }^{b} F\right\|_{\mathscr{L}_{t, r}^{q^{\prime}} H_{\omega}^{s-1 / 2}} .\right.\right.\right.\right.
$$

The proof of theorems above is essentially the same as the proof of [13, Theorem 3.1]. We notice that $H^{s}\left(S^{n-1}\right)$-valued analogs of [13, Lemmas 3.5, 3.6] hold.

The following proposition is important for the estimate of the nonlinear term.

Proposition 3.8. Let $n \geq 2$ and let $p>1$. Let $f$ satisfy $(\mathrm{H})$. Let $s_{0}, s, p$ satisfy $s \geq s_{0}, p>s_{0}, s>$ $(n-1) / 2$. Then,

$$
\begin{gathered}
\|f(u)\|_{H^{s_{0}\left(S^{n-1}\right)} \leq C\|u\|_{H^{s}\left(S^{n-1}\right)}^{p}}, \\
\|f(u)-f(v)\|_{L^{2}\left(S^{n-1}\right)} \leq C\left(\|u\|_{H^{s}\left(S^{n-1}\right)}^{p-1}+\|v\|_{H^{s}\left(S^{n-1}\right)}^{p-1}\right)\|u-v\|_{L^{2}\left(S^{n-1}\right)} .
\end{gathered}
$$

Proof of Proposition 3.8. The estimate (3.13) follows from the Moser type estimate

$$
\|f(u)\|_{H^{s_{0}\left(S^{n-1}\right)}} \leq C\|u\|_{L^{\infty}\left(S^{n-1}\right)}^{p-1}\|u\|_{H^{s_{0}\left(S^{n-1}\right)}}
$$

with $p>\max \left(s_{0}, 1\right)$ and the Sobolev embedding (3.5). For the proof of the Moser type estimate, see (4.5) in Appendix. The estimate (3.14) follows from the Hölder inequality and also the Sobolev embedding (3.5). 
Proof of Theorem 3.2. We first define the successive sequence $\left\{u_{j}\right\}$ by

$$
\begin{gathered}
u_{j}(t)=u_{0}(t)+\int_{0}^{t}(-\Delta)^{-1 / 2} \sin \left[(t-s)(-\Delta)^{1 / 2}\right] f\left(u_{j-1}(s)\right) d s, \\
u_{0}(t)=\varepsilon\left(\cos \left[t(-\Delta)^{1 / 2}\right] \phi+(-\Delta)^{-1 / 2} \sin \left[t(-\Delta)^{1 / 2}\right] \psi\right) .
\end{gathered}
$$

We observe that $u_{j}(\lambda t, \lambda x)=\lambda^{-2 /(p-1)} u_{j}(t, x)$ holds inductively for $j \geq 0$ by the homogeneity of $\phi, \psi$, and $f$. Note that this fact enables us to apply Theorems 3.6, 3.7.

By the triangle inequality we have

$$
\begin{aligned}
\left\|\left.\left|t^{2}-\right| x\right|^{2}\left|{ }^{\gamma} u_{j}\left\|_{\mathscr{L}_{t, r}^{p+1} H_{\omega}^{s}} \leq C\right\|\right| t^{2}-\left.|x|^{2}\right|^{\gamma} u_{0}\right\|_{\mathscr{L}_{t, r}^{p+1} H_{\omega}^{s}} & \\
& +C\left\|\left.\left.\left|t^{2}-\right| x\right|^{2}\right|^{\gamma} \int_{0}^{t}(-\Delta)^{-\frac{1}{2}} \sin \left[(t-s)(-\Delta)^{\frac{1}{2}}\right] f\left(u_{j-1}(s)\right) d s\right\|_{\mathscr{L}_{t, r}^{p+1} H_{\omega}^{s}},
\end{aligned}
$$

where $\gamma=1 /(p-1)-(n+1) / 2(p+1), s=(n-1) / 2+\delta$ with $\delta>0$.

As for the first term on the right hand side of (3.15), we apply Theorem 3.5 with $\alpha=2 /(p-1)$, $q=p+1$. Then, the assumptions of Theorem 3.5 are satisfied for $p_{0}(n)<p<(n+3) /(n-1)$ and we have

$$
C\left\|\left.\left.\left|t^{2}-\right| x\right|^{2}\right|^{\gamma} u_{0}\right\|_{\mathscr{L}_{t, r}^{p+1} H_{\omega}^{s}}=C_{0} \varepsilon .
$$

In fact, $(n-1) / 2<\alpha<\min ((n-1) / 2, n-1)$ is equivalent to $\max ((n+5) /(n+1),(n+1) /(n-1))$, and $1-(\alpha+2) /(n+1)<1 / q<1-\alpha /(n-1)$ is equivalent to $p_{0}(n)<p<(n+3) /(n-1)$.

As for the estimate of the second term on the right hand side of (3.15), we first consider the case where $2 \leq n \leq 4$. In this case, we apply Theorem 3.6 with $\alpha=2 /(p-1), q=p+1$. Then, the assumptions of Theorem 3.6 are satisfied for $p_{0}(n)<p<(n+3) /(n-1)$ and we have

$$
\begin{aligned}
& C\left\|\left.\left.\left|t^{2}-\right| x\right|^{2}\right|^{\gamma} \int_{0}^{t}(-\Delta)^{-\frac{1}{2}} \sin \left[(t-s)(-\Delta)^{\frac{1}{2}}\right] f\left(u_{j-1}(s)\right) d s\right\|_{\mathscr{L}_{t, r}^{p+1} H_{\omega}^{s}} \\
& \leq C\left\|\left.\left.\left|t^{2}-\right| x\right|^{2}\right|^{p \gamma} f\left(u_{j-1}\right)\right\|_{\mathscr{L}_{t, r}^{(p+1) / p} H_{\omega}^{s}} \\
& \leq C\left\|\left.\left.\left|t^{2}-\right| x\right|^{2}\right|^{\gamma} u_{j-1}\right\|_{\mathscr{L}_{t, r}^{p+1} H_{\omega}^{s}}^{p} .
\end{aligned}
$$

It is worth noting that $\alpha<(n-1) / q^{\prime}$ is equivalent to $p>p_{0}(n)$. In the last inequality above, we used Proposition 3.8 with $s=s_{0}$ and $p>p_{0}(n)$. Note that

$$
p_{0}(n)>\frac{n-1}{2} \text { for } 2 \leq n \leq 4 \text {. }
$$

Therefore, we obtain

$$
\left\|\left.\left.\left|t^{2}-\right| x\right|^{2}\right|^{\gamma} u_{j}\right\|_{\mathscr{L}_{t, r}^{p+1} H_{\omega}^{s}} \leq C_{0} \varepsilon+\left.\left.C||\left|t^{2}-\right| x\right|^{2}\right|^{\gamma} u_{j-1} \|_{\mathscr{L}_{t, r}^{p+1} H_{\omega}^{s}}^{p} .
$$

We next consider the case $n=5$. In this case, we apply Theorem 3.7 with $\alpha=2 /(p-1), q=p+1$. Then, the assumptions of Theorem 3.7 are satisfied for $p_{0}(n)<p<(n+3) /(n-1)$ and we have

$$
\begin{aligned}
& C\left\|\left.\left.\left|t^{2}-\right| x\right|^{2}\right|^{\gamma} \int_{0}^{t}(-\Delta)^{-\frac{1}{2}} \sin \left[(t-s)(-\Delta)^{\frac{1}{2}}\right] f\left(u_{j-1}(s)\right) d s\right\|_{\mathscr{L}_{t, r}^{p+1} H_{\omega}^{s}} \\
& \leq C\left\|\left.\left.\left|t^{2}-\right| x\right|^{2}\right|^{p \gamma} f\left(u_{j-1}\right)\right\|_{\mathscr{L}_{t, r}^{(p+1) / p} H_{\omega}^{s-1 / 2}} \\
& \leq C\left\||| t^{2}-\left.|x|^{2}\right|^{\gamma} u_{j-1}\right\|_{\mathscr{L}_{t, r}^{p+1} H_{\omega}^{s}}^{p} .
\end{aligned}
$$


We notice that $q>4(n-1) /(2 n-3)$ is always satisfied when $p>p_{0}(n)$. In the last inequality above, we used Proposition 3.8 with $s_{0}=s-1 / 2$. In fact, since

$$
p_{0}(5)>s_{0}=s-\frac{1}{2}=\frac{3}{2}+\delta \text { for } n=5,
$$

we have

$$
\left\|f\left(u_{j-1}\right)\right\|_{H^{s-1 / 2}\left(S^{n-1}\right)} \leq C|| u_{j-1} \|_{H^{s}\left(S^{n-1}\right)}^{p},
$$

for $p>p_{0}(5)$. Note that $p_{0}(5)=(3+\sqrt{17}) / 4(\fallingdotseq 1.75)$. Therefore, we also obtain (3.16). Thus, in each case, we are able to prove

$$
\left\|\left.\left.\left|t^{2}-\right| x\right|^{2}\right|^{\gamma} u_{j}\right\|_{\mathscr{L}_{t, r}^{p+1} H_{\omega}^{s}} \leq 2 C_{0} \varepsilon
$$

inductively. Applying the second inequality in Proposition 3.8, we also obtain

$$
\left\|\left.\left.\left|t^{2}-\right| x\right|^{2}\right|^{\gamma}\left(u_{j+1}-u_{j}\right)\right\|_{\mathscr{L}_{t, r}^{p+1} L_{\omega}^{2}} \leq C \varepsilon^{p-1}\left\|\left.\left.\left|t^{2}-\right| x\right|^{2}\right|^{\gamma}\left(u_{j}-u_{j-1}\right)\right\|_{\mathscr{L}_{t, r}^{p+1} L_{\omega}^{2}}
$$

Therefore, we conclude that $\left\{u_{j}\right\}$ converges to the solution $u$ satisfying (3.4).

\section{Appendix}

In this appendix we summarize basic properties of Sobolev spaces over the unit sphere. The main purpose of this section is to show the Moser type estimates (4.5), and to show the equivalence of the definitions of the Sobolev spaces by (2.2) and by the Bessel potentials (4.10).

For $m \geq 1$, let $M$ be a connected $m$-dimensional compact Riemannian manifold furnished with a smooth Riemannian metric $g$. Let $\left\{\left(U_{j}, \exp _{j}^{-1}\right)\right\}_{1 \leq j \leq J}$ be the local charts of $M$ with the exponential maps $\left\{\exp _{j}\right\}_{1 \leq j \leq J}$. Let $\left\{\psi_{j}\right\}_{1 \leq j \leq J}$ be the resolution of unity with supp $\psi_{j} \subset U_{j}$ for $1 \leq j \leq J$. The Triebel-Lizorkin spaces $F_{p q}^{s}(M)$ on $M$ are defined by

$$
F_{p q}^{s}(M)=\left\{u \in \mathscr{D}^{\prime}(M) \mid\left\|u ; F_{p q}^{s}(M)\right\| \equiv \sum_{j=1}^{J}\left\|\left(\psi_{j} u\right) \circ \exp _{j} ; F_{p q}^{s}\left(\mathbb{R}^{m}\right)\right\|<\infty\right\}
$$

for $1 \leq p<\infty, 1 \leq q \leq \infty$, or $p=q=\infty$, and $-\infty<s<\infty$, where $\mathscr{D}^{\prime}(M)$ denotes the distributions on $M$ (see (27) in [33]). The above definition depends on the resolution of unity, but the spaces defined by another resolution of unity have equivalent norms. For the definition of $F_{p q}^{s}\left(\mathbb{R}^{m}\right)$, see [32, §2.3.1].

The Sobolev spaces $H^{s, p}(M)$ for $1<p<\infty$ and $-\infty<s<\infty$ are defined by the Laplace-Beltrami operator $\Delta_{M}$. We have relations

$$
H^{s, p}\left(\mathbb{R}^{m}\right)=F_{p, 2}^{s}\left(\mathbb{R}^{m}\right), \quad H^{s, p}(M)=F_{p, 2}^{s}(M)
$$

for $1<p<\infty$ and $-\infty<s<\infty$ (see the theorem in [32, §1.5.1], and (38) in [33]). As a simple result, the embedding $H^{s, p}\left(\mathbb{R}^{m}\right) \hookrightarrow L^{\infty}\left(\mathbb{R}^{m}\right)$ for $1<p<\infty$ and $s>m / p$ yields the embedding

$$
H^{s, p}(M) \hookrightarrow L^{\infty}(M) \text { for } s>m / p, 1<p<\infty
$$


Indeed, since $\left\{\psi_{j}\right\}_{1 \leq j \leq J}$ is the resolution of unity, we have

$$
\left\|u ; L^{\infty}(M)\right\| \leq \sum_{j=1}^{J}\left\|\left(\psi_{j} u\right) \circ \exp _{j} ; L^{\infty}\left(\mathbb{R}^{m}\right)\right\| .
$$

The embedding $H^{s, p}\left(\mathbb{R}^{m}\right) \hookrightarrow L^{\infty}\left(\mathbb{R}^{m}\right)$ and (4.2) yields the bound

$$
\left\|\left(\psi_{j} u\right) \circ \exp _{j} ; L^{\infty}\left(\mathbb{R}^{m}\right)\right\| \leq C\left\|\left(\psi_{j} u\right) \circ \exp _{j} ; F_{p 2}^{s}\left(\mathbb{R}^{m}\right)\right\|
$$

So that (4.3) follows from (4.1) and (4.2).

Next we consider the Moser type estimates for nonlinearities on $M$.

We introduce the notation $N(s, p)$ as follows. For $s \geq 0$ and $1 \leq p<\infty$, we say that $f$ satisfies $N(s, p)$ if $f \in C^{[s]}\left(\mathbb{R}^{2}, \mathbb{C}\right), f(0)=\cdots=f^{([s])}(0)=0$,

$$
\left|f^{([s])}(z)-f^{([s])}(w)\right| \leq \begin{cases}C(|z|+|w|)^{p-[s]-1}|z-w| & \text { if }[s]+1 \leq p, \\ C|z-w|^{p-[s]} & \text { if } s<p<[s]+1, \\ 0 & \text { if } p \leq s,\end{cases}
$$

where the derivative is understood in terms of $\partial / \partial z$ and $\partial / \partial \bar{z}$ with the identification $\mathbb{C} \simeq \mathbb{R}^{2}$ (see also $[8,23])$.

Lemma 4.1. Let $1 \leq p<\infty, s \geq 0$ and $f$ satisfy $N(s, p)$. Then

$$
\left\|f(u) ; H^{s}(M)\right\| \leq C\left\|u ; L^{\infty}(M)\right\|^{p-1}\left\|u ; H^{s}(M)\right\| .
$$

Proof of Lemma 4.1. The estimate (4.5) follows immediately for $s=0$. We assume $s>0$ in the following. By (4.1) and (4.2), we have

$$
\left\|f(u) ; H^{s}(M)\right\| \leq C \sum_{j=1}^{J}\left\|\left(\psi_{j} f(u)\right) \circ \exp _{j} ; H^{s}\left(\mathbb{R}^{m}\right)\right\| .
$$

Let $\left\{\tilde{\psi}_{j}\right\}_{1 \leq j \leq J}$ be functions with $\tilde{\psi}_{j} \geq 0, \tilde{\psi}_{j}=1$ on supp $\psi_{j}$, and supp $\tilde{\psi}_{j} \subset U_{j}$. The support condition on $\tilde{\psi}_{j}$ and the Leibniz rule (see Lemma A4 in [15]) give us

$$
\begin{aligned}
& \left\|\left(\psi_{j} f(u)\right) \circ \exp _{j} ; H^{s}\left(\mathbb{R}^{m}\right)\right\|=\left\|\left(\psi_{j} f\left(\tilde{\psi}_{j} u\right)\right) \circ \exp _{j} ; H^{s}\left(\mathbb{R}^{m}\right)\right\| \\
& \leq C\left\|\psi \circ \exp _{j} ; H^{s, p_{1}}\left(\mathbb{R}^{m}\right)\right\|\left\|f\left(\left(\tilde{\psi}_{j} u\right) \circ \exp _{j}\right) ; L^{p_{2}}\left(\mathbb{R}^{m}\right)\right\| \\
& \quad+C\left\|\psi \circ \exp _{j} ; L^{\infty}\left(\mathbb{R}^{m}\right)\right\|\left\|f\left(\left(\tilde{\psi}_{j} u\right) \circ \exp _{j}\right) ; H^{s}\left(\mathbb{R}^{m}\right)\right\|,
\end{aligned}
$$

where $p_{2} \equiv 2+\varepsilon, p_{1} \equiv(4+2 \varepsilon) / \varepsilon$ with $\varepsilon>0$ sufficiently small. Applying the embedding $H^{s}\left(\mathbb{R}^{m}\right) \hookrightarrow$ $L^{p_{2}}\left(\mathbb{R}^{m}\right)$ to the second factor in the first term on the RHS of the last inequality, we have

$$
\left\|\left(\psi_{j} f(u)\right) \circ \exp _{j} ; H^{s}\left(\mathbb{R}^{m}\right)\right\| \leq C\left\|f\left(\left(\tilde{\psi}_{j} u\right) \circ \exp _{j}\right) ; H^{s}\left(\mathbb{R}^{m}\right)\right\|,
$$

where the constant $C$ may depend on $\psi_{j}$ and $\exp _{j}$. The well-known Moser type estimates (see (2.3) in [17] for example) give us

$$
\left\|f\left(\left(\tilde{\psi}_{j} u\right) \circ \exp _{j}\right) ; H^{s}\left(\mathbb{R}^{m}\right)\right\| \leq C\left\|\left(\tilde{\psi}_{j} u\right) \circ \exp _{j} ; L^{\infty}\left(\mathbb{R}^{m}\right)\right\|^{p-1}\left\|\left(\tilde{\psi}_{j} u\right) \circ \exp _{j} ; H^{s}\left(\mathbb{R}^{m}\right)\right\| .
$$


Let $\left\{\phi_{j}\right\}_{1 \leq j \leq J}$ be the resolution of unity defined by $\phi_{j} \equiv\left(\sum_{1 \leq k \leq J} \tilde{\psi}_{k}\right)^{-1} \tilde{\psi}_{j}$. The Leibniz rule and the embedding $H^{s}\left(\mathbb{R}^{m}\right) \hookrightarrow L^{p_{2}}\left(\mathbb{R}^{m}\right)$ yields

$$
\left\|\left(\tilde{\psi}_{j} u\right) \circ \exp _{j} ; H^{s}\left(\mathbb{R}^{m}\right)\right\| \leq C\left\|\left(\phi_{j} u\right) \circ \exp _{j} ; H^{s}\left(\mathbb{R}^{m}\right)\right\| .
$$

So that by the bound

$$
\left\|\left(\tilde{\psi}_{j} u\right) \circ \exp _{j} ; L^{\infty}\left(\mathbb{R}^{m}\right)\right\| \leq C\left\|u ; L^{\infty}(M)\right\|,
$$

(4.1) and (4.2), we obtain (4.5) as required.

Let $\left(x_{1}, \cdots, x_{m}\right)$ be the local coordinate system for some coordinate neighborhood on $M$. And let $g_{i j}$, $1 \leq i, j \leq m$, be the components of $g$ in terms of $\left(x_{1}, \cdots, x_{m}\right)$. For any tangent vector $X=\sum_{j=1}^{m} X_{j} \partial / \partial x_{j}$, and any smooth function $h$ on $M$, the operators $\operatorname{div}_{M}$ and $\operatorname{grad}_{M}$ are defined by

$$
\operatorname{div}_{M} X \equiv \frac{1}{\sqrt{\operatorname{det}\left(g_{i j}\right)}} \sum_{k=1}^{m} \frac{\partial}{\partial x_{k}}\left(\sqrt{\operatorname{det}\left(g_{i j}\right)} X_{k}\right), \quad \operatorname{grad}_{M} h \equiv \sum_{1 \leq i, j \leq m} g^{i j} \frac{\partial h}{\partial x_{j}} \frac{\partial}{\partial x_{i}},
$$

where $\left(g^{i j}\right)$ is the inverse matrix of $\left(g_{i j}\right)$.

Let us consider the case $M=S^{m}$. Let $g$ be the Riemannian metric on $S^{m}$ induced from the standard metric in $\mathbb{R}^{m+1}$. We take a local chart $(U, \psi)$ with $U=\left\{\left(x_{1}, \cdots, x_{m+1}\right) \in S^{m} ; x_{m+1}>0\right\}, \psi\left(x_{1}, \cdots, x_{m+1}\right)=$ $\left(x_{1}, \cdots, x_{m}\right)$. By a simple calculation, we have the expression of $g$ on the local chart $U$ such as

$$
g_{i j}=\delta_{i j}+\frac{x_{i} x_{j}}{x_{m+1}^{2}}, \quad g^{i j}=\delta_{i j}-x_{i} x_{j}, \quad \operatorname{det}\left(g_{i j}\right)=x_{m+1}^{-2},
$$

where $x_{m+1}=\sqrt{1-x_{1}^{2}-\cdots-x_{m}^{2}}$ and $\delta_{i j}$ is Kronecker's delta. By a direct calculation, we have the relation

$$
\begin{aligned}
\left(\Delta_{S^{m}} h(r \cdot)\right)(\omega) & =\left(\operatorname{div}_{S^{m}} \operatorname{grad}_{S^{m}} h(r \cdot)\right)(\omega) \\
& =\sum_{1 \leq k<\ell \leq m+1}\left(\left(x_{k} \partial_{x_{\ell}}-x_{\ell} \partial_{x_{k}}\right)^{2} h\right)(r \omega), \quad x=r \omega, \quad r>0, \quad \omega \in S^{m}
\end{aligned}
$$

for any $h \in C^{2}\left(\mathbb{R}^{m+1}\right)$. So that we obtain the well-known formula

$$
\Delta_{\mathbb{R}^{m+1}}=\frac{\partial^{2}}{\partial r^{2}}+\frac{m}{r} \frac{\partial}{\partial r}+\frac{1}{r^{2}} \Delta_{S^{m}}
$$

Let $\mathscr{H}_{k}^{m}$ be the space of spherical harmonics of degree $k$ on $S^{m}$. For any $Y^{k} \in \mathscr{H}_{k}^{m}, Y^{k}$ satisfies

$$
-\Delta_{S^{m}} Y^{k}=k(k+m-1) Y^{k}
$$

by the properties $\Delta_{\mathbb{R}^{m+1}}\left(Y^{k}(r \omega)\right)=0, Y^{k}(r \omega)=r^{k} Y^{k}(\omega)$ and (4.8). We recall the definition of Sobolev spaces on manifolds. Since the Bessel potentials $\left(1-\Delta_{S^{m}}\right)^{-s / 2}$ with $s>0$ can be defined in $L^{2}\left(S^{m}\right)$ via the spectrum theorem, the Sobolev norms on $S^{m}$ are defined by

$$
\left\|u ; H^{s}\left(S^{m}\right)\right\| \equiv \begin{cases}\left\|\left(1-\Delta_{S^{m}}\right)^{s / 2} u ; L^{2}\left(S^{m}\right)\right\| & \text { for } s \geq 0, \\ \left\|\left(1-\Delta_{S^{m}}\right)^{-j} u ; H^{s+2 j}\left(S^{m}\right)\right\| & \text { for } s<0 \text { with } s+2 j>0\end{cases}
$$

(see Definition 4 in [33, p312]). So that the norms defined by (2.2) and (4.10) are equivalent for even integers $s$ by (4.9), for nonnegative real numbers $s$ by the interpolation argument, and also for negative real numbers $s$ by the definition in (4.10). Therefore we can use the embeddings (4.3) and the Moser type estimates (4.5) for the norm defined by (2.2). 


\section{References}

[1] N. Arcozzi, X. Li, Riesz transforms on spheres, Math. Res. Lett. 4 (1997), 401-412.

[2] J. Bergh, J. Löfström, “Interpolation Spaces,” Springer-Verlag (1976).

[3] N. Burq, F. Planchon, J. G. Stalker, A. S. Tahvildar-Zadeh, Strichartz estimates for the wave and Schrödinger equations with the inverse-square potential, J. Funct. Anal. 203 (2003), 519-549.

[4] P. L. Butzer, H. Berens, “Semi-Groups of Operators and Approximation,” Springer-Verlag (1967).

[5] P. D’ Ancona, V. Georgiev, H. Kubo, Weighted decay estimates for the wave equation, J. Differential Equations 177 (2001), 146-208.

[6] G. Freud, "Orthogonal Polynomials,” Pergamon, Oxford, New York (1971).

[7] V. Georgiev, H. Lindblad, C. D. Sogge, Weighted Strichartz estimates and global existence for semilinear wave equations, Amer. J. Math. 119 (1997), 1291-1319.

[8] J. Ginibre, T. Ozawa, G. Velo, On the existence of the wave operators for a class of nonlinear Schrödinger equations, Ann. Inst. H. Poincaré Phys. Thoér. 60 (1994), 211-239.

[9] K. Hidano, Scattering and self-similar solutions for the nonlinear wave equations, Differential Integral Equations 15 (2002), 405-462.

[10] D. Jerison, C. E. Kenig, Unique continuation and absence of positive eigenvalues for Schrödinger operators. With an appendix by E. M. Stein, Ann. of Math. (2) 121 (1985), 463-494.

[11] F. John, Blow-up of solutions of nonlinear wave equations in three space dimensions, Manuscripta Math. 28 (1979), 235-268.

[12] J. Kato, T. Ozawa, On solutions of the wave equation with homogeneous Cauchy data, Asymptotic Anal. 37 (2004), 93-107.

[13] J. Kato, T. Ozawa, Weighted Strichartz estimates and existence of self-similar solutions for semilinear wave equations, Indiana Univ. Math. J. 52 (2003), 1615-1630.

[14] J. Kato, T. Ozawa, Weighted Strichartz estimates for the wave equation in even space dimensions, Math. Z. 247 (2004), 747-764.

[15] T. Kato, On nonlinear Schrödinger equations. II. $H^{s}$-solutions and unconditional well-posedness, J. Anal. Math. 67 (1995), 281-306.

[16] O. Kavian, F. B. Weissler, Finite energy self-similar solutions of a nonlinear wave equation, Comm. Partial Differential Equations 15 (1990), 1381-1420.

[17] N. Kita, T. Wada, Sharp asymptotic behavior of solutions to nonlinear Schrödinger equations in one space dimension, Funkcial. Ekvac. 45 (2002), 53-69. 
[18] H. Kubo, K. Kubota, Asymptotic behaviors of radially symmetric solutions of $\square u=|u|^{p}$ for super critical values $p$ in even space dimensions, Japan. J. Math. 24 (1998), 191-256.

[19] H. Lindblad, C. D. Sogge, Long-time existence for small amplitude semilinear wave equations, Amer. J. Math. 118 (1996), 1047-1135.

[20] S. Machihara, M. Nakamura, K. Nakanishi, T. Ozawa, Endpoint Strichartz estimates and global solutions for the nonlinear Dirac equation, J. Funct. Anal. 219 (2005), 1-20.

[21] Y. Meyer, Large-time behavior and self-similar solutions of some semilinear diffusion equations, Harmonic analysis and partial differential equations (Chicago, 1996), Chicago Lectures in Math., Univ. Chicago Press (1999), 241-261.

[22] C. Müller, "Analysis of Spherical Symmetries in Euclidean Spaces," Applied Mathematical Sciences 129, Springer-Verlag, New York (1998).

[23] M. Nakamura, T. Ozawa, Small data scattering for nonlinear Schrödinger, wave and Klein-Gordon equations, Ann. Sc. Norm. Super. Pisa Cl. Sci. 1 (2002), 435-460.

[24] H. Pecher, Self-similar and asymptotically self-similar solutions of nonlinear wave equations, Math. Ann. 316 (2000), 259-281.

[25] H. Pecher, Sharp existence results for self-similar solutions of semilinear wave equations, NoDEA Nonlinear Differential Equations Appl. 7 (2000), 323-341.

[26] F. Ribaud, A. Youssfi, Global solutions and self-similar solutions of semilinear wave equation, Math. Z. 239 (2002), 231-262.

[27] C. D. Sogge, Oscillatory integrals and spherical harmonics, Duke Math. J. 53 (1986), 43-65.

[28] E. M. Stein, G. Weiss, Fractional integrals on $n$-dimensional Euclidean space, J. Math. Mech. 7 (1958), 503-514.

[29] E. M. Stein, G. Weiss, "Introduction to Fourier Analysis on Euclidean Spaces," Princeton Mathematical Series 32, Princeton University Press, Princeton (1971).

[30] H. Takamura, Blow-up for semilinear wave equations with slowly decaying data in high dimensions, Differential Integral Equations 8 (1995), 647-661.

[31] D. Tataru, Strichartz estimates in the hyperbolic space and global existence for the semilinear wave equation, Trans. Amer. Math. Soc. 353 (2001), 795-807.

[32] H. Triebel, "Theory of function spaces. II," Monographs in Mathematics, 84, Birkhäuser Verlag, Basel, 1992.

[33] H. Triebel, Spaces of Besov-Hardy-Sobolev type on complete Riemannian manifolds, Ark. Mat. 24 (1986), 299-337. 
Jun Kato

JSPS Research Fellow.

Department of Mathematics, Kyoto University, Kyoto 606-8502, Japan.

E-mail : jkato@math.kyoto-u.ac.jp

Makoto Nakamura

Graduate School of Information Sciences, Tohoku University, Sendai 980-8579, Japan.

E-mail:m-nakamu@math.is.tohoku.ac.jp

Tohru Ozawa

Department of Mathematics, Hokkaido University, Sapporo 060-0810, Japan. 